\title{
Energy dependence of $\bar{K} N$ interaction in nuclear medium
}

\author{
Aleš Cieplý
}

Received: date / Accepted: date

\begin{abstract}
When the $\bar{K} N$ system is submerged in nuclear medium the $\bar{K} N$ scattering amplitude and the final state branching ratios exhibit a strong energy dependence when going to energies below the $\bar{K} N$ threshold. A sharp increase of $\bar{K} N$ attraction below the $\bar{K} N$ threshold provides a link between shallow $\bar{K}$-nuclear potentials based on the chiral $\bar{K} N$ amplitude evaluated at threshold and the deep phenomenological optical potentials obtained in fits to kaonic atoms data. We show the energy dependence of the in-medium $K^{-} p$ amplitude and demonstrate the impact of energy dependent branching ratios on the $\Lambda$-hypernuclear production rates.
\end{abstract}

Keywords kaon-nucleon amplitude $\cdot$ nuclear medium · hypernuclei

A key issue in studying in-medium $K^{-}$meson interactions concerns the strength of the attractive $K^{-}$nuclear potential. Chirally based coupled channels calculations lead to $K^{-}$nuclear optical potentials that are about $80 \mathrm{MeV}$ deep at nuclear densities $\rho=\rho_{0}=0.17 \mathrm{fm}^{-3}$. The incorporation of kaon selfenergy reduces the depth of the potential to approximately $40-50 \mathrm{MeV}$ while much deeper potentials, in the range $\operatorname{Re} V_{K^{-}}\left(\rho_{0}\right) \sim-(150-200) \mathrm{MeV}$ are obtained in comprehensive global fits to kaonic atoms data [1]. In our recent paper [2] we reported on a new self consistent treatment of chirally motivated $K^{-} N$ amplitudes that lead to deep $K^{-}$nuclear potentials, considerably deeper than the 'shallow' potentials deduced in earlier models based on the chiral amplitude [3, 4].

The elementary $\bar{K} N$ interaction at threshold and low energies is well understood within chiral models combined with the coupled channels re-summation techniques. In our approach we employ chirally motivated coupled-channel

A. Cieplý

Nuclear Physics Institute, 25068 Řež, Czech republic

Tel.: +420-266173284

Fax: +420-220940165

E-mail: cieply@ujf.cas.cz 

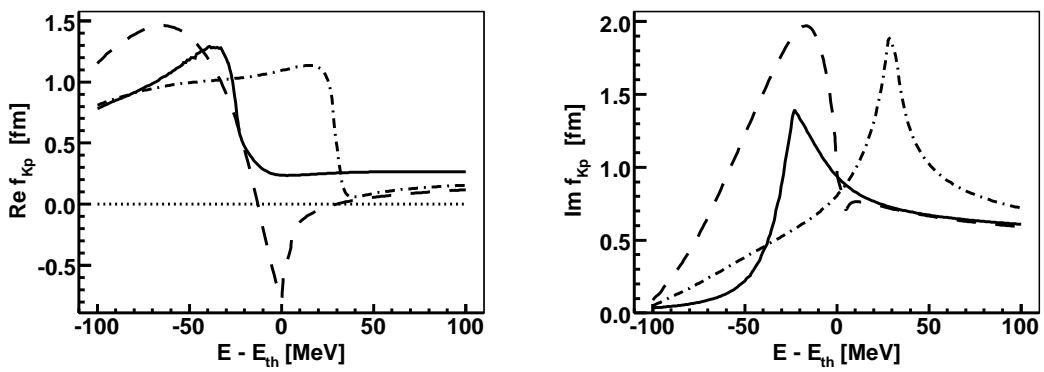

Fig. 1 Energy dependence of the $K^{-} p$ scattering amplitude. The left and right panels refer to the real and imaginary parts of the amplitude, respectively, and $E_{t h}$ denotes the $K^{-} p$ threshold energy. Dashed curves: free space, dot-dashed: Pauli blocking in nuclear medium at $\rho=\rho_{0}$, solid curves: combined effect of Pauli blocking and hadron selfenergies at $\rho=\rho_{0}$.

potentials that are taken in a separable form [5]. The meson-baryon channels are composed from the $\pi \Lambda, \pi \Sigma, \bar{K} N, \eta \Lambda, \eta \Sigma$, and $K \Xi$ states (taken with all appropriate charge combinations). The parameters of the model, the inverse ranges that define the off-shell Yamaguchi form factors and low energy constants of the SU(3) chiral Lagrangian that contribute to the inter-channel couplings, are fitted to the kaonic hydrogen and low energy $K^{-} p$ reactions data. A detailed description of our model can be found in Refs. [5] and [2].

When the $\bar{K} N$ system is submerged in nuclear medium the $\bar{K} N$ scattering amplitude and the final state branching ratios exhibit a strong energy dependence when going to energies below the $\bar{K} N$ threshold. In Fig. 11 we show an energy dependence of the elastic scattering amplitudes $f_{K^{-}}$in free space and for two versions of in-medium modifications, performed with a leading order chiral model labeled as TW1 in Ref. 2. The free space $K^{-} p$ amplitude exhibits a typical structure related to an $I=0 \bar{K} N$ quasi-bound state which is assigned to a well known $\Lambda(1405)$ resonance. When the $K^{-} p$ system is submerged in nuclear medium the Pauli blocking shifts the structure to higher energies, about $30-40 \mathrm{MeV}$ above the $\bar{K} N$ threshold. However, the addition of kaon selfenergies (we also included baryon and pion selfenergies in our model, though their impact is minute) brings it back below the threshold as demonstrated by the solid curves in the figure. In this regime the most striking feature is a sharp increase in the real part of the amplitude when going to subthreshold energies. Consequently, the $K^{-} p$ interaction becomes much stronger at energies about $30 \mathrm{MeV}$ below the $K^{-} p$ threshold with respect to its strength at threshold. We have shown 2 that this is exactly the region of energies probed by kaons at the lowest $K$-atomic orbits, so the $K^{-}$-nuclear optical potential becomes much deeper than when it were constructed from the amplitudes taken at the $\bar{K} N$ threshold.

The impact of the in-medium $\bar{K} N$ dynamics and energy dependence of the $\bar{K} N$ amplitudes on the characteristics of kaonic atoms and kaon-nuclear states was investigated in Ref. 2]. Here we mention another application to the $\Lambda$ hypernuclear production in the $\left(K_{\text {stop }}^{-}, \pi^{-}\right)$reactions. Recently, the production 

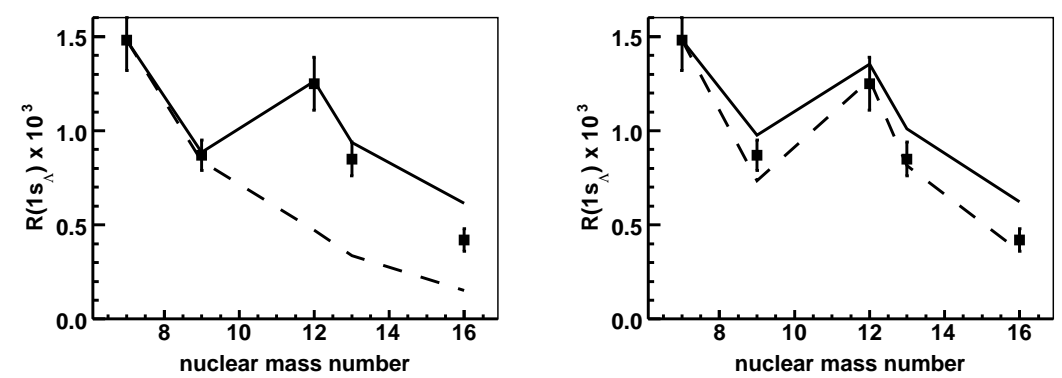

Fig. 2 The $A$-dependence of the $1 s_{\Lambda}$ hypernuclear formation rates, experimental data from Ref. 6. The theoretical rates are normalized to the ${ }^{7} \mathrm{Li}$ experimental value and were calculated with a phenomenological density dependent kaon-nuclear optical potential (dashed lines) and with the chirally motivated $K^{-}$-nuclear optical potential (solid lines). Left panel: elementary $\operatorname{BR}\left(K^{-} N \rightarrow \pi \Lambda\right)$ fixed at the threshold value for nuclear density $\rho=\rho_{0} / 2$, right panel: energy and density dependent BR.

rates were established by the FINUDA collaboration 6 for five $p$-shell nuclear targets from ${ }^{7} \mathrm{Li}$ to ${ }^{16} \mathrm{O}$. Since the absolute normalization of the experimental capture rates is a delicate matter and the calculated rates are generally much lower than the measured ones, we have focused on the $A$-dependence of the $1 s_{\Lambda}$ formation rates 7 . The Figure 2 shows the effect of energy dependent branching ratios $\mathrm{BR}\left(K^{-} n \rightarrow \pi^{-} \Lambda\right)$ on the rates computed within the framework of the distorted wave impulse approximation. There, the $K^{-}$-nuclear optical potential is used to generate the $K$-atomic initial state wave function. In the left panel (which was not published earlier) of Fig. 2 we present the rates calculated with the BR fixed at a value obtained at the $\bar{K} N$ threshold while the theoretical rates in the right panel (taken from Ref. [7]) were calculated with energy dependent BR averaged over the region of relevant subthreshold energies, specific for each nuclear target. With the BR fixed at the $\bar{K} N$ threshold the $A$-dependence is reproduced much better by the chirally motivated optical potential which is shallow at the $\bar{K} N$ threshold. Since both the phenomenological and chirally motivated optical potentials are sufficiently deep at the subthreshold energies relevant for the evaluation of the energy dependent BR, they lead to similar $1 s_{\Lambda}$ formation rates.

Acknowledgements The author acknowledges a fruitful collaboration with E. Friedman, A. Gal, D. Gazda, J. Mareš, V. Krejčiřík and J. Smejkal who coauthored the papers the report is based on. This work was supported by the GACR Grant No. 202/09/1441.

\section{References}

1. E. Friedman, A. Gal, and C.J. Batty, Nucl. Phys. A579, 518 (1994)

2. A. Cieplý, E. Friedman, A. Gal, D. Gazda, and J. Mareš, Phys. Rev. C84, 045206 (2011)

3. T. Waas, N. Kaiser, and W. Weise, Phys. Lett. B365, 12 (1996); ibid. B379, 34 (1996)

4. A. Ramos and E. Oset, Nucl. Phys. A671, 481 (2000)

5. A. Cieplý and J. Smejkal, Eur. Phys. J. A43, 191 (2010)

6. M. Agnello et al. [FINUDA Coll.], Phys. Lett. B698, 219 (2011); ibid. B622, 35 (2005)

7. A. Cieplý, E. Friedman, A. Gal, and V. Krejčiř́ík, Phys. Lett. B698, 226 (2011) 